\title{
EL IMPACTO DE LA NOCIÓN REPUBLICANA DE LIBERTAD SOBRE EL ROL DEL ABOGADO
}

THE IMPACT OF THE REPUBLICAN NOTION OF FREEDOM ON THE LAWYER'S ROLE

\section{Hugo Omar Seleme*}

Resumen: El objetivo del presente trabajo es analizar las características que adquiere el rol de abogado una vez que se adopta la concepción republicana de libertad como no dominación. La metodología empleada es el análisis conceptual y el equilibrio reflexivo. En la sección II se muestra cómo el modo dominante de concebir el rol de abogado se encuentra fundado en una concepción alternativa de libertad como no-interferencia. En la sección III se presentan las críticas que los republicanos han dirigido a esta concepción de libertad y se presenta a la concepción superadora de libertad como nodominación. Finalmente se muestran las nuevas características que adopta el rol de abogado si se entiende a la libertad como no-dominación. Primero, desaparece la tensión entre el carácter del abogado como auxiliar de la justicia y como defensor de la libertad del cliente. Segundo, el abogado deja de ser un mero asistente técnico y pasa a ser la primera barrera que evita que el cliente se vuelva un agente dominador. Tercero, el sistema adversarial pasa a ser un instrumento para dispersar el control entre las partes, evitando que una domine a la otra. Cuarto, la auto-regulación de la profesión se vuelve un mecanismo para evitar la dominación horizontal y vertical.

Palabras clave: Libertad como no-dominación, libertad como nointerferencia, ética profesional, celosa defensa, responsabilidades conflictivas

\footnotetext{
* Catedrático de Ética, Facultad de Derecho, Universidad Nacional de Córdoba (Argentina). Investigador del CONICET. Director de la Maestría en Derecho y Argumentación Director del Programa de Ética y Teoría Política. hugoseleme@gmail.com
} 
Abstract: The objective of this paper is to analyze the characteristics the lawyer's role acquires once the republican conception of freedom as nondomination is adopted. Two methods are used, conceptual analysis and reflective equilibrium. Section II shows how the dominant conception of lawyer's role is based on an alternative idea of freedom as non-interference. In section III, the criticisms that Republicans have directed to this conception of freedom are presented. Finally, the new features the lawyer's role adopts once this defective conception of freedom is replaced are listed and explained. First, the tension between the character of the lawyer as an auxiliary to justice and as a defender of the client's freedom disappears. Second, the lawyer ceases to be a mere technical assistant and becomes the first barrier that prevents the client from becoming a dominating agent. Third, the adversarial system becomes an instrument to disperse control between the parties, preventing one from dominating the other. Fourth, the self-regulation of the profession becomes a mechanism to avoid horizontal and vertical domination.

Keywords: Freedom as Non-Domination, Freedom as Non-Interference, Legal Ethics, Zealous Defense, Conflictive Responsibilities

Sumario. I. Introducción. II. Libertad como no interferencia y abogacía. III. Libertad como no Dominación y Abogacía. IV. Conclusión. Referencias.

\section{INTRODUCCIÓN}

En nuestras sociedades, caracterizadas por la asunción de valores liberales, el rol de abogado se encuentra indefectiblemente ligado a la protección de la libertad. Su imagen ideal es la de alguien comprometido con la celosa defensa de la libertad de su cliente. Los abogados se perciben a sí mismos como la primera barrera de contención frente a los intentos de avasallamiento de los derechos y libertades de quienes representan o asesoran.

Este ideal ha sido incorporado a los códigos deontológicos. La manera más común de hacerlo ha consistido en traducir la protección de la libertad del cliente en términos referidos a la promoción de sus intereses. Así lo hacía, por ejemplo, el Model Code of Professional Responsibility de 1974 de la American Bar Association (en adelante "ABA"), que en su consideración ética 7-1 establecía que el abogado debía promover "celosamente" los intereses y objetivos de su cliente. Las Model Rules que lo sucedieron reemplazaron el "celo" por la "diligencia" y la "prontitud", 
pero mantuvieron el lenguaje de los intereses. La "celosa defensa", sin embargo, vuelve a hacer su aparición en el comentario 3 a la regla 1.3 donde se sostiene que el abogado debe actuar «con celo a la hora de abogar en beneficio de su cliente».

La misma traducción de la defensa de la libertad en términos de promoción de intereses se encuentra en otras regulaciones deontológicas. En Argentina, el Código de Ética para el ejercicio de la profesión de abogado de la Capital Federal señala en su art. 19.a que el abogado debe «atender los intereses confiados con celo, saber y dedicación». Una exigencia análoga incorpora el Código de Ética y Disciplina de La Pampa en su art.1 que establece en relación con los abogados «que la esencia de su deber profesional es consagrarse enteramente a los intereses de su cliente, y poner en la defensa de los derechos del mismo su celo, saber y habilidad».

El art. 3 del Código de Ética Profesional del Colegio de Abogados de Chile, por su parte, estipula que «el abogado debe obrar siempre en el mejor interés de su cliente y anteponer dicho interés al de cualquier otra persona, incluyendo al suyo propio». El Código de Ética de los Abogados para Perú establece en su art. 27 que «(e)s deber del abogado defender el interés del cliente de manera diligente y con un elevado estándar de competencia profesional». El Código de Ética del Ilustre y Nacional Colegio de Abogados de México se refiere a la protección de la libertad y a la promoción de los intereses, como si fuesen equivalentes. Su preámbulo declara que en la relación que lo vincula con su cliente, el abogado «tiene la obligación de defender sus derechos y libertades; es, por lo tanto, el asesor y defensor de su cliente». El art. 2.7. 1, estipula que «(e)l Abogado tiene la obligación de defender lo mejor posible los intereses de su cliente, incluso en contraposición a los suyos propios, a los de un colega o a aquellos de la profesión en general». Estipulaciones semejantes se encuentran en el Código de Ética Profesional de la Barra Mexicana. Según este plexo normativo la esencia del deber profesional del abogado «es defender diligentemente y con estricto apego a las normas morales, los derechos de su cliente» (art. 1) sin “anteponer su propio interés al de su cliente» (art. 11.2).

Junto con la defensa de la libertad del cliente, otro rasgo aparece como distintivo del ejercicio profesional: el abogado debe tener en miras el interés público caracterizado como respeto a la ley o promoción de la justicia. Las regulaciones deontológicas también han incorporado esta exigencia. El preámbulo de las Model Rules of Professional Conduct de la ABA señala que el abogado es un «officer of the legal system».

En Argentina, el Código de Ética para el ejercicio de la profesión de Abogado en la Capital Federal establece que «(e)s misión esencial de la abogacía el afianzar la justicia» (art. 6) y que «(e)s deber del abogado 
preservar y profundizar el Estado de Derecho» (art. 7). El art. 1 del Código de Ética y Disciplina de La Pampa estipula que el abogado «es un servidor de la justicia y un colaborador de su administración». La normativa que regula el ejercicio profesional en la provincia de Córdoba, por su parte, establece que es deber del abogado «(p)restar su asistencia profesional como colaborador del Juez y en servicio de la Justicia» (art. $19 \S 1$ ).

El Código de Ética Profesional chileno en su art. 2 señala que «(l)as actuaciones del abogado deben promover, y en caso alguno afectar (...) la correcta y eficaz administración de justicia, y la vigencia del estado de derecho». El Código de Ética del Abogado de Perú dice en su art. 2 que la profesión de abogado «(c)umple una función social al servicio del Derecho y la Justicia» y el art. 3 agrega que «(l)a abogacía tiene por fin la defensa de los derechos de las personas y la consolidación del Estado de Derecho, la justicia y el orden social». El Código de Ética del Ilustre y Nacional Colegio de Abogados de México en su art. 2.7.2, luego de señalar que «(e)l abogado podrá defender a su cliente, aún a sabiendas de que es culpable, y procurar obtener una sentencia de inocencia», establece un límite: «siempre y cuando no recurra a ningún medio ilícito». En su preámbulo declara que la misión del abogado «no se limita a ejecutar fielmente un mandato en el marco del Derecho. En un Estado de Derecho, el Abogado es indispensable para lograr el respeto y cumplimiento de la Justicia». El Código de Ética Profesional de la Barra Mexicana, sostiene que la defensa de los intereses del cliente debe hacerse «en el marco de la ley de la forma que considere más apropiada» (art. 7.2). Su preámbulo agrega que «(e)l abogado ha de tener presente que es un servidor del derecho y un coadyuvante de la justicia».

La manera en que estas dos exigencias son interpretadas (la de proteger la libertad del cliente y la de respetar la ley o promover la justicia) depende, como es obvio, del modo en que se caracterice a la libertad. La visión dominante en los códigos deontológicos según la cual proteger la libertad es equivalente a promover los intereses del cliente presupone concebir a la libertad de una manera concreta: como ausencia de interferencias a las conductas deseadas. Dicho de manera simple, si alguien puede hacer lo que desea entonces es libre. Este modo de concebir la libertad hunde sus raíces hasta Thomas Hobbes y se contrapone con una idea de libertad más antigua - la republicana - que puede rastrearse al menos hasta el pensamiento clásico greco-romano. De acuerdo con esta concepción republicana de libertad alguien es libre cuando habita un esquema institucional que establece protecciones que lo vuelve inmune frente al poder arbitrario o incontrolado de interferencia de otros ciudadanos o del propio Estado. 
Mientras Isahia Berlin (1969) ha sido el principal defensor contemporáneo de la posición que sostiene que la libertad debe concebirse como no-interferencia, Philip Pettit (1997) y Quentin Skinner (1995) han sido los impulsores de una corriente conocida como neorepublicanismo que afirma que la libertad debe concebirse como ausencia de dominación ${ }^{1}$. Este ha sido uno de los debates más activos en el ámbito de la filosofía política durante los últimos años. El objetivo del presente trabajo es mostrar los contornos que adquiere la profesión de abogado una vez que la libertad es interpretada como proponen los republicanos.

Para alcanzar este objetivo, en la sección II se muestra cómo, si se concibe a la libertad como no-interferencia, el rol de abogado adquiere contornos específicos que coinciden con la visión dominante de la profesión. Según esta visión la exigencia de defender la libertad del cliente y la de ser un auxiliar de la justicia se encuentran en tensión. El carácter adversarial del sistema jurídico se presenta, entonces, como un modo de resolver el conflicto haciendo que la defensa de los intereses privados de los clientes redunde en el interés común por la justicia. El preámbulo de las Model Rules of Professional Conduct señala que «when an opposing party is well represented, a lawyer can be a zealous advocate on behalf of a client and at the same time assume that justice is being done». La tarea del abogado es meramente técnica y debe brindar cualquier interpretación del derecho que sea funcional a los intereses del propio cliente, despreocupándose por el carácter legítimo o ilegítimo de la norma y por el hecho de que la interpretación propuesta fruste los fines que persigue. La misma técnica interpretativa se traslada a los estándares éticos que regulan el ejercicio profesional.

En la sección III se presentan, en primer lugar, las razones que los republicanos han ofrecido para dejar de concebir a la libertad como nointerferencia y pasar a entenderla como libertad como no-dominación. En segundo lugar, se muestra cómo adoptar esta concepción de libertad conduce a otra manera de concebir el rol de abogado. El carácter conflictivo de la exigencia de respeto a la ley o promoción de la justicia, por un lado, y la de protección de la libertad del cliente, por el otro, se aminora o desaparece. La tarea del abogado deja de ser meramente técnica. Es parte fundamental de la tarea profesional preguntarse por el carácter legítimo de las normas e interpretarlas de modo que sus fines no se vean frustrados. La protección de la libertad del cliente no equivale a promover sus intereses, cualquiera que

${ }^{11}$ La lista de pensadores enrolados en el neorepublicanismo es extensa y continúa en aumento. Entre ellos cabe mencionar a Frank Lovett (2001, 2010, 2012), Maurizio Viroli (2002), John Maynor (2003), Cecile Laborde (2008) y a José Luis Martí (2006). 
estos sean, sino sólo aquellos que no son contrarios a las normas legítimas. Finalmente, aparece un nuevo modo de concebir las exigencias éticas que regulan la profesión y los mecanismos para garantizar su cumplimiento.

\section{LIBERTAD COMO NO-INTERFERENCIA Y ABOGACÍA}

Isahia Berlin presenta a la libertad no-interferencia como una variante corregida de la libertad no-frustración propuesta por Thomas Hobbes. Para este último la libertad sólo exige la inexistencia de obstáculos —naturales o fruto de la interferencia deliberada de otro agente - para poder llevar adelante la opción preferida por el agente en cuestión (Pettit, 2011, p. 697). Berlin propone corregir esta noción en dos puntos cruciales. En primer lugar, sólo la interferencia fruto de la acción deliberada de un agente cuenta como una transgresión a la libertad ${ }^{2}$. En segundo lugar, para que alguien sea libre no deben existir obstrucciones deliberadas —esto es interferencias - a la opción preferida en el mundo actual y en los mundos posibles cercanos donde las preferencias del agente son diferentes y hasta opuestas. De acuerdo con la reconstrucción de la posición de Berlin ofrecida por Pettit, alguien es libre en el mundo actual «not just in virtue of its being a world without interference but also in virtue of its being a world where certain features mean that you would not suffer interference even if you chose other than you actually did» $(2011$, p. 701$)$.

Según Philip Pettit (2011, p. 699), las razones por las que Berlín propone abandonar la libertad como no-frustración hobbesiana radican en que parece contraintuitivo afirmar que alguien puede volverse libre sólo por extinguir los deseos que tiene de realizar aquellos cursos de acción que se encuentran interferidos. Si ser libre sólo consistiese en poder hacer cualquier cosa que uno desease sin sufrir la interferencia de otros, tal como sostiene la libertad como no-frustración, un modo de alcanzar la libertad consistiría en eliminar todos aquellos deseos que no pueden ser satisfechos debido a la interferencia de terceros. Como esta conclusión parece absurda, ya que no diríamos que alguien que actúa de este modo es alguien libre sino más bien alguien resignado, Berlín concluye que la libertad como no-frustración es errónea y debe ser reemplazada por la libertad como no-interferencia.

\footnotetext{
${ }^{2}$ «You lack political liberty or freedom only if you are prevented from attaining a goal by human beings» (Berlin, 2002, p. 169). Este es un punto con el que Pettit acuerda, para quien:

«Natural limitations reduce the range over which you can enjoy your freedom from the will of others and may even be instrumental in making you vulnerable to that will - and on those grounds they will call for remedy. But according to these theories, such limitations do not in themselves take away from your freedom; they do not make you unfree in the way that other agents can do so (...)» (2011, p. 694).
} 
A pesar de sus divergencias, la libertad como no-interferencia comparte con la libertad como no-frustración la manera de ver el derecho. Dado su carácter coercitivo, todas las normas jurídicas en mayor o menor medida interfieren con la realización de ciertas conductas individuales. El Derecho es siempre una restricción, o una "cadena", «even if it protects you from being bound in chains that are heavier than those of the law, say arbitrary despotism and chaos (...)» (Berlin, 2002, p. 170). Sin importar cuál sea la forma de gobierno, o el procedimiento de creación y aplicación de las normas jurídicas, o su carácter legítimo o ilegítimo, toda norma jurídica interfiere con la libertad individual. La libertad como no-interferencia no toma distancia de las palabras de Hobbes (1997) en relación con la inscripción libertas en la torre de la ciudad de Luca: «no man can thence inferre, that a particular man has more Libertie, or Immunitie from the service of the Commonwealth there, than in Constantinople. Whether a Commonwealth be Monarchical, or Popular, the Freedome is still the same» (p. 132).

$\mathrm{Si}$ se emplea esta idea de libertad como no-interferencia para dar contenido a las dos exigencias que definen el rol de abogado - la de proteger la libertad del cliente y la de actuar con apego a la ley, promoviendo la realización de la justicia - aparece una visión conflictualista que pone a ambas exigencias en tensión ${ }^{3}$. Adicionalmente, alrededor de esta tensión, y de la noción de libertad como no-interferencia que la justifica, aparecen otros rasgos distintivos del rol profesional, a saber: la idea de que el abogado cumple meramente una tarea de asistencia técnica para que el cliente satisfaga sus intereses, la confianza de que al defender el interés privado del cliente contribuirá a la realización del interés público y la justicia, el recelo frente a cualquier regulación estatal sobre la conducta profesional, y la percepción de la ética profesional como una "ética de principios o reglas". Veamos cada uno de estos puntos en detalle.

En primer lugar, si se concibe a la libertad como no-interferencia es fácil dar contenido a la idea de que el abogado, en tanto promotor de los intereses del cliente, es un defensor de su libertad. Caracterizar la protección de los intereses o deseos del cliente en términos de libertad es algo sencillo cuando la libertad es interpretada como la posibilidad de llevar adelante sin interferencias los cursos de acción que coinciden con los deseos o preferencias que uno tiene o podría tener. El abogado, al intentar que el cliente pueda actuar tal y como lo desea, o al intentar minimizar las interferencias que existen sobre sus conductas, se transforma en un garante de su libertad individual.

${ }^{3}$ En esta sección sigo, con ligeras modificaciones, lo que he desarrollado en la obra "Libertad como no-interferencia y Abogacía" (inédita). 
En segundo lugar, la libertad como no-interferencia también permite dar contenido a la exigencia de que el abogado sea "auxiliar de la justicia", traduciendo este requerimiento por promover la justicia en mero "respeto por la legalidad". Es natural traducir el aspecto público que posee el rol de abogado en términos de mera legalidad — haciendo que la "justicia" de la que el abogado es auxiliar no tenga vinculación alguna con la legitimidad política o moral - cuando se sostiene que toda norma jurídica, sea o no legítimo su origen, es igualmente costosa en términos de libertad. Si el derecho es siempre una "cadena", las diferencias entre las leyes legítimas y las ilegítimas se diluye, y queda abierto el camino para que la preocupación de los abogados por el interés público sea interpretada en términos de mero respeto a la ley sea cual sea su origen o contenido.

Entender a la libertad como no-interferencia hace que el problema de la legitimidad o la justicia desaparezcan del horizonte profesional. El abogado deja de percibirse como un genuino "auxiliar de la justicia" que debe ejercitar la profesión con una mirada puesta en la corrección de los resultados, y pasa a ser un colaborador de la "administración de justicia", esto es del Poder Judicial. En tanto "auxiliar de la justicia" el abogado solamente soporta la exigencia de no transgredir la ley o no contribuir a que su cliente la transgreda.

Aparece, así, la manera actualmente dominante de combinar las exigencias que pesan sobre el abogado. Por un lado, éste debe proteger la libertad de su cliente, promoviendo sus intereses, utilizando todos los medios legales a su alcance. El límite a la defensa es la mera legalidad. Esta combinación conduce de manera directa a la idea de la "celosa defensa dentro de los límites de la ley", muy presente en tantos códigos deontológicos. Dado que ambas exigencias están en tensión, debido a que toda ley tiene costos en términos de libertad individual, el abogado debe extremar los recursos argumentativos e interpretativos para disminuir el impacto que las normas jurídicas puedan tener en la satisfacción de los intereses de su cliente. Es decir, debe promover o defender los intereses de su cliente brindando la interpretación de la ley que sea más favorable a ellos.

La tarea del abogado, definida con estos contornos, no requiere preguntarse cuáles son los fines que persigue la norma jurídica ni, menos aún, intentar promoverlos. La promoción de los intereses colectivos corresponde al legislador, no al abogado. Son aquéllos quienes deben debatir qué fines colectivos son dignos de ser perseguidos y cuáles son los mejores medios legales para alcanzarlos. La razón de esta desconexión entre la tarea del abogado y la promoción de los fines de las normas jurídicas, tiene que ver con la independencia conceptual que existe entre la libertad como nointerferencia y la libertad como auto-gobierno colectivo o auto-control. 
Para quienes conciben a la libertad como no-interferencia la libertad corporizada en la actividad legislativa es una de índole positiva, la misma que está relacionada con la pregunta «What, or who, is the source of control or interference that can determine someone to do, or be, this rather than that?»(Berlin, 2002, p. 169). En una democracia la mayor parte de la ciudadanía, a través de sus representantes, son la fuente que determina los fines que persigue el derecho y controlan su poder de interferencia. Esto, sin embargo, no aminora la amenaza que las normas jurídicas ejercen sobre la libertad como no-interferencia de los ciudadanos, de la cual los abogados son garantes.

Que las normas jurídicas sean fruto del ejercicio de la libertad positiva de la comunidad política, no dice nada todavía sobre el grado de libertad negativa como no-interferencia que gozan los ciudadanos. Ambos conceptos de libertad responden a dos preguntas diferentes. Según Berlín mientras la libertad positiva responde a la pregunta "¿quién me gobierna?", la libertad negativa se interroga por "¿qué tanto el gobierno me interfiere?" (Berlin, 2002, p. 178).

Berlin enfatiza la independencia entre ambos tipos de libertad al señalar la posibilidad de que la libertad como no-interferencia puede ser promovida por un gobierno autocrácito. Tal sería el caso del déspota «who leaves his subjects a wide area of liberty may be unjust, or encourage the, wildest inequalities, care little for order, or virtue, or knowledge» (Berlin, 2002, p. 176). De esto concluye que la libertad como no-interferencia «is not at any rate logically, connected with democracy or self-government» (Berlin, 2002, p. 176). Por este motivo es igualmente posible que los gobiernos democráticos la vulneren.

De acuerdo con Berlin (2002, p. 191), quienes conciben a la libertad como auto-control individual, y sostienen que ser libre consiste en que las partes más valiosas de uno mismo se impongan sobre las menos valiosas, tarde o temprano pasan a aplicar este concepto positivo de libertad como auto-gobierno al ámbito político. Al hacer esta transición del auto-gobierno individual al colectivo, terminan justificando que los fines determinados por la porción más valiosa de la comunidad política — sea que ésta se conciba de modo aristocrático como la más educada, o de modo democrático como la más numerosa - sean impuestos coercitivamente sobre aquellos que no los comparten. De este modo, concluye, «what had begun as a doctrine of freedom turned into a doctrine of authority and, at times, of oppression, and became the favored weapon of despotism» (p. 37) ${ }^{4}$.

\footnotetext{
${ }^{4}$ Es necesario aclarar que el potencial justificatorio del despotismo del concepto de libertad positiva entendida como auto-gobierno colectivo no es exclusivo de éste. El propio Berlin
} 
Esta desconfianza frente al auto-gobierno colectivo se traslada al modo de concebir la actividad política. Si el primero es concebido como un ejercicio de libertad positiva, esto es como "libertad para", es natural concebir a la segunda como un intento de imponer una determinada forma de vida. La política se transforma en un modo de ejercitar "la libertad para imponer una forma de vivir", adoptando tintes perfeccionistas potencialmente peligrosos para la libertad como no-interferencia de los ciudadanos.

En síntesis, concebir al autogobierno colectivo como un tipo de libertad positiva y a la libertad individual como libertad negativa, da sustento a la idea de que la protección de la libertad del cliente, y la promoción de sus intereses que debe llevar adelante el abogado, no está relacionada con la promoción de los intereses colectivos vinculados con el auto-gobierno y corporizados en el ordenamiento jurídico. No es tarea del abogado intentar promover los fines que la comunidad política intenta alcanzar a través de las normas jurídicas. Aun si estos fines han sido determinados de manera democrática, nada garantiza que su promoción sea congruente con la protección de la libertad individual. Si los fines que persigue la comunidad política son una potencial amenaza para la libertad individual, no es necesario que el abogado interprete los límites jurídicos teniéndolos en mente, ni que se preocupe por promoverlos.

Si se concibe a la libertad como no-interferencia, existe un único fin valioso que pueden tener las normas, sea o no fruto del auto-gobierno colectivo: limitar la interferencia. Éstas, por lo tanto, deben ser interpretadas como protecciones frente a la interferencia de terceros, incluida la interferencia estatal. Hasta los derechos que podrían estar más vinculados con el autogobierno, como los derechos políticos, deben ser entendidos de este modo. Su finalidad no consiste en permitir que los ciudadanos participen en la conducción de los asuntos colectivos, sino que actúan como «safeguards against intervention by the State» (Berlin, 2002, p. 171).

Esta última consideración explica por qué el abogado, en tanto defensor de la libertad de su cliente, debe, por un lado, apegarse estrictamente a lo que prescriben las normas jurídicas y, por el otro, esforzarse por interpretarlas de manera que no interfieran con los deseos que aquél posee. Si las normas jurídicas deben ser vistas como salvaguardas frente a la interferencia — incluida la del Estado - el abogado que no se apega a ellas contribuye a socavarlas poniendo en riesgo la libertad

(2002, p. 37) se encarga de señalar que justificar ese tipo de gobiernos opresivos podría haber sido logrado utilizando la idea de libertad negativa. Sin embargo, señala, aunque esta posibilidad existe, no ha sido el camino que tomó el pensamiento político en occidente. 
individual que debe proteger. Sin embargo, si las normas jurídicas valiosas desde el punto de la libertad como no-interferencia son aquellas que tienen por objeto poner freno a la interferencia estatal, el abogado debe interpretar todo el entramado jurídico presuponiendo que éste es el fin que persigue, sean cuales sean los fines específicos que el legislador tuvo en mente a la hora de dictarlas.

Lo señalado conduce a un modo de entender la función del abogado dentro del sistema adversarial. La protección de la libertad como nointerferencia de cada uno de los ciudadanos se alcanza a través de una especie de división del trabajo. Cada abogado sólo debe defender celosamente los intereses de su cliente "dentro de los límites de la ley". El sistema adversarial articula esta división del trabajo y permite alcanzar la aspiración expresada en el preámbulo de las Model Rules of Professional Conduct: la celosa defensa de partes opuestas permite que la justicia sea satisfecha. Si la libertad es concebida como mera no-interferencia, el proceso judicial se transforma en un juego de suma cero en el que lo que una parte gana en términos de libertad es equivalente a lo que la otra pierde. La justicia no puede medirse teniendo en cuenta el resultado - que siempre tendrá el mismo valor en términos de libertad como no-interferencia- sino sólo teniendo en cuenta la equidad del procedimiento. De allí la idea de que, si ambas partes cuentan con igualdad de armas para intentar satisfacer sus intereses a través del proceso judicial, y ambas cuentan con una celosa defensa por parte de su abogado, entonces la justicia ha sido satisfecha.

La idea de la "celosa defensa dentro de los límites de la ley", entendida con el trasfondo de la libertad como no-interferencia, conduce a un rasgo adicional del rol de abogado. El abogado sólo brinda a su cliente el conocimiento técnico del que dispone para que éste pueda satisfacer sus intereses. Su rol es meramente instrumental. Los fines que el abogado persigue en el ejercicio profesional son los del cliente y no es su tarea moldearlos o evaluarlos. El abogado que disminuye la interferencia de la que es objeto su cliente, simplemente haciendo que éste altere sus fines o deseos, no se ha comportado como un promotor de su libertad. Como se recordará, justamente éste último es el punto de crítica que Berlin formula a la libertad como no-frustración hobbesiana.

Para que la libertad del cliente sea respetada, el abogado debe encargarse de extinguir las obstrucciones deliberadas —incluidas las corporizadas en las normas jurídicas de carácter coercitivo- no sólo en relación con las conductas que se propone llevar adelante su cliente dado los deseos que de hecho tiene, sino también en relación con aquellas conductas que acometería si tuviese deseos diferentes u opuestos. De manera que provocar la alteración de los deseos del cliente para que ahora sean 
congruentes con la norma jurídica, no es un modo de promover su libertad. Por supuesto, cuando los deseos del cliente son ilegales el abogado no puede contribuir a su satisfacción, dado el componente público de su rol profesional. Pero aun en este caso, intentar alterar los deseos del cliente no es una exigencia que se siga de su carácter de protector de la libertad individual, ya que esta alteración no aumenta en modo alguno la libertad como no-interferencia de la que aquél goza. Mientras mayor sea la dependencia del abogado en relación con los deseos del cliente, mayor garantía existe de que actúe como una salvaguarda a la libertad como nointerferencia de éste.

Concebir a la libertad como no-interferencia, a la política y a la injerencia estatal como potencialmente peligrosa, y a las normas jurídicas como resguardos frente a la interferencia de terceros, conduce también a un modo de entender los estándares que regulan la conducta profesional. Adiestrados en interpretar a las normas jurídicas de manera restrictiva, intentando minimizar su poder de interferencia sobre la conducta del cliente, no es de extrañar que los abogados entiendan de este mismo modo los estándares normativos que regulan su propia conducta. Un abogado bien adiestrado en temas de ética profesional es aquel que conoce a la perfección las exigencias de los códigos de ética, y se apega escrupulosamente a su letra, a la vez que es hábil para encontrar interpretaciones que sin, vulnerar la literalidad de las normas, se desentienden por completo de su espíritu y finalidad.

La "infraestructura ética" que han desarrollado los grandes estudios jurídicos, construyendo departamentos poblados por especialistas en ética profesional, ha estado focalizada en evitar incurrir en responsabilidad civil por prácticas contrarias a los estándares éticos ${ }^{5}$. Estos departamentos actúan en relación con el resto de los abogados a los que aconsejan, del mismo modo y con las mismas herramientas interpretativas que éstos actúan con sus clientes. Así como el abogado intenta minimizar el poder de interferencia de las normas jurídicas sobre las conductas del cliente, los departamentos de ética profesional intentan minimizar la interferencia de los estándares éticos sobre la conducta de los abogados, evitando de este modo su incursión en causales de responsabilidad.

\footnotetext{
${ }^{5}$ Elizabeth Chambliss y David Wilkins (2002) estuvieron entre los primeros en advertir la necesidad de esta "infraestructura ética" así como su surgimiento embrionario dentro de algunas grandes firmas. Una de sus conclusiones es que los encargados de regular la profesión judicial están de acuerdo en que es necesario moverse «beyond the individualistic paradigm that currently dominates professional regulation and to focus on the development of self-regulatory structures within firms» (p. 716).
} 
El recelo de la política y de la injerencia estatal, asociada con la libertad como no-interferencia, brinda razones adicionales para la resistencia de los abogados frente a cualquier intento estatal de regular la profesión. Este recelo se emparenta con el ideal de auto-regulación a través de los colegios profesionales. El principal rol de los Colegios de Abogados pasa a ser el de resistir cualquier interferencia del Estado en el ejercicio profesional. El ideal de que la profesión se auto-regule minimizando las regulaciones de origen estatal se justifica como una manera de garantizar la independencia de los abogados frente al Estado. En este sentido son interpretadas declaraciones como la del preámbulo de las Model Rules de la ABA que sostiene que «(a)n independent legal profession is an important force in preserving government under law, for abuse of legal authority is more readily challenged by a profession whose members are not dependent on government for the right to practice».

De esta manera la dependencia del abogado en relación con los deseos y preferencias del cliente se combina con la independencia de los abogados, a la hora de ejercer la profesión, de las regulaciones estatales. Siguiendo esta línea de pensamiento la ABA ha ido tan lejos a la hora de defender la autoregulación, como para sugerir que cualquier intento de reglar el ejercicio profesional que no provenga de una norma expresa dictada por el Congreso, debe ser respetuosamente desobedecida por los abogados. Así, por ejemplo, una declaración del Congreso de Delegados de la ABA en 1975 señalaba que

«(C)ualquier principio legal que (...) permita u obligue a un abogado a revelar información confidencial debería ser establecido únicamente por una norma dictada luego de que se hayan considerado de manera exhaustiva y cuidadosa los intereses públicos involucrados y debería ser resistida a menos que la revelación fuese requerida de manera clara por la ley».

Las opiniones y comentarios a las Model Rules también reflejan esta idea de noble resistencia a la regulación de la profesión por parte del Estado (Koniak \& Cohen, 2000, p. 182). La idea detrás de esta afirmación es que idealmente la conducta profesional sólo debería ser regulada por el colegio profesional y que los abogados deben abandonar ese ideal sólo cuando existe una norma estatal expresa cuya literalidad no puede ser evitada de manera alguna.

Otra variante del ideal de auto-regulación es aquél que se realiza a través de los instrumentos del mercado. Existe una línea argumental que conduce desde la libertad como no-interferencia a la justificación del mercado como herramienta de control. El mercado no es más que el intercambio voluntario, libre de interferencias, de bienes y servicios. El caso de los servicios profesionales, se sostiene, no es una excepción, y lo que 
debe garantizar el Estado son los mismos extremos que deben ser garantizados en la transacción de cualquier otro bien o servicio. Siendo esto así, la regulación que protege a los consumidores es suficiente para garantizar el funcionamiento del mercado de los servicios legales. No es necesaria ninguna regulación específica — dictada por los órganos estatales o por los colegios profesionales - y cualquier intento adicional de reglar la conducta profesional es una vulneración a la libertad como no-interferencia. Esta defensa del mercado en base a la libertad como no-interferencia es completada con un argumento de eficiencia: cualquier intento de regulación centralizada será necesariamente inferior a la organización espontánea que surge del mercado ${ }^{6}$.

Adicionalmente, si se concibe a la libertad como no-interferencia la ética profesional comienza a estar focalizada en identificar y evitar los conflictos de intereses, tal como sucede actualmente. Si el abogado es el defensor de la libertad del cliente, y ésta es entendida como la posibilidad del cliente de conducirse de acuerdo con sus intereses actuales o potenciales, es lógico que se vea como un problema de suma gravedad la posibilidad de que el abogado responda a intereses diferentes a los de su cliente. Si el abogado representa otros intereses - incluidos los suyos propios- no existe ninguna garantía de que actúe como protector de la libertad de aquél.

Finalmente, en el paradigma que concibe a la libertad como nointerferencia la ética profesional es vista como una "ética de principios" en lugar de una "ética de virtudes". La razón de la resistencia a concebir al buen abogado como aquel que posee ciertos rasgos de carácter valiosos, en lugar de aquel que simplemente sigue las leyes, es la vinculación que se plantea, por un lado, entre la idea de virtud y la noción de libertad positiva y, por el otro, entre ésta y el totalitarismo y perfeccionismo. La noción de virtud corporiza la idea de que existen ciertas partes de nosotros mismos que son más valiosas que otras y que las primeras deben gobernar a las últimas. La prudencia es más valiosa que la temeridad, el coraje más valioso que la cobardía, la misericordia es superior a la indiferencia, etc. Esta idea de gobierno de las partes superiores sobre las inferiores no se diferencia de la noción de libertad positiva. Según Berlín, cuando se hace el traslado del

\footnotetext{
${ }^{6}$ Hayek (1973, p. 51) ha sido el principal defensor de este tipo de posiciones. Según su visión cualquier sistema de planificación central tendrá un enorme problema para recolectar información porque la información se encuentra dispersa y es difícil de adquirir. Un punto central en toda la obra de Hayek, que por supuesto se aplica a la regulación del ejercicio de la abogacía, es que el orden puede surgir de manera espontánea. Hayek (1960, p. 59) veía con cierta frustración que la refutación de la falacia que había conducido en las ciencias naturales de constatar la existencia de orden a postular la existencia de un supremo diseñador, no hubiese tenido su réplica en las ciencias sociales.
} 
auto-gobierno individual al colectivo, se termina justificando la idea de que la porción supuestamente más valiosa de la sociedad ejercite el control sobre las menos valiosas y, en segundo lugar, que lo haga para imponer un ideal humano dotado de ciertos rasgos de carácter.

Ahora bien, si esta es la visión que tienen de la virtud aquellos que conciben a la libertad como no-interferencia, no es de extrañar que vean con reticencia cualquier intento de controlar el ejercicio profesional a través de la promoción en los abogados de ciertos rasgos de carácter que se consideran valiosos $^{7}$. La idea de que estos rasgos valiosos o virtudes van a ser identificados por la porción moralmente más elevada de la profesión, se les aparece como una puerta de ingreso al totalitarismo. Adicionalmente, que estos rasgos vayan a ser desarrollados a través de un programa de enseñanza o entrenamiento - implementado, por ejemplo, en las escuelas de derecholes parece un caso intolerable de perfeccionismo. Frente a esta alternativa, la idea de una ética profesional de reglas o principios, centrada en las conductas y no en el carácter, surge como más segura y libre de riesgos.

La vinculación que he trazado entre, por un lado, la desconfianza que los defensores de la libertad negativa tienen de la libertad positiva y, por el otro, la desconfianza de la apelación a la virtud en el ejercicio profesional, no debe verse como excluyente de otros nexos. Como no podría ser de otro modo, los vasos comunicantes que existen entre la concepción negativa de libertad y el abandono del ideal de jurista virtuoso son múltiples, y la desconfianza en la libertad positiva es sólo uno de ellos. Otro nexo tiene que ver con que tradicionalmente los jueces y abogados se preparaban para resolver los casos difíciles. Para hacerlo debían desarrollar la virtud de la sabiduría práctica que consistía en «a subtle and discriminating sense of how the (often conflicting) generalities of legal doctrine should be applied in concrete disputes» (Kronman, 1995, p. 21) La misma visión tenían los profesores de derecho que veían a su profesión como un ejercicio de sabiduría práctica que tenía por objeto contribuir a la misma tarea que llevaban adelante los abogados y jueces.

Esta visión, señala Kronman (1995, p. 175), cambió con un cultor de la libertad negativa: Thomas Hobbes. A partir de Hobbes el derecho dejó de ser visto como una rama de la sabiduría práctica y pasó a ser considerado

\footnotetext{
${ }^{7}$ A nivel contemporáneo se distinguen, al menos, cuatro variantes de éticas de la virtud: eudemonistas (Annas, 2011), basadas en el agente o ejemplares (Slote, 2001; Zagzebski, 2010), centradas en objetivos (Swanton, 2003) y platónicas (Chappell, 2014). A los fines del presente texto no es necesario tomar partido por ninguna de ellas. Basta la noción genérica de virtud como rasgo de carácter valioso compartida por todas ellas.
} 
como un conjunto de reglas ${ }^{8}$. Consecuentemente, la tarea de los profesores de derecho dejó de ser percibida como una complementaria con la tarea de jueces y abogados. El estudio del derecho dejó de estar focalizado en el ejercicio y desarrollo de una virtud y pasó a ser una ciencia focalizada en el conocimiento de normas. Si el derecho no es más que un conjunto de reglas cuya aplicación sólo requiere conocimiento, la tarea que realizan los profesores de derecho es una especie de ciencia, esto es un tipo de conocimiento teórico que no requiere ningún tipo de sabiduría práctica o virtud. Consecuentemente, un buen abogado es simplemente aquel que conoce las reglas - incluidas las que se aplican a su propia conducta - y las sigue; del mismo modo que el buen juez es aquel que conoce las reglas jurídicas y las aplica. Todo rasgo de carácter es borrado por completo de la escena ${ }^{9}$.

\section{LIBERTAD COMO NO-DOMINACIÓN Y ABOGACÍA}

Dado el carácter central que la libertad ocupa en la configuración del rol de abogado, no es de extrañar que los defectos de la primera tengan impacto en la manera en que se delinea el segundo. El neo-republicanismo ha llamado la atención sobre las falencias que posee la libertad como nointerferencia y ha propuesto una concepción alternativa que concibe a la libertad como no-dominación. Una vez que se adopta esta concepción republicana de libertad el rol profesional adopta nuevos contornos. A delinearlos está dedicada la presente sección.

Como he señalado, el argumento de Berlin en contra de la libertad como no-frustración hobbesiana afirma que es contraintuitivo sostener que puede alcanzarse la libertad meramente por extinguir los deseos de realizar cursos de acción sobre los que existen interferencias. Si lo contrario a la libertad es la frustración, uno puede evitar la frustración y alcanzar la libertad meramente por acomodar los propios deseos en lugar de eliminar las interferencias (Pettit, 2011, p. 699).

\footnotetext{
${ }^{8}$ Aunque como señala John Finnis (1996) esta idea puede rastrearse hasta el Trasímaco de Platón y encuentra cabida luego en el pensamiento medieval su resurgimiento en el pensamiento contemporáneo se debe a Thomas Hobbes.

${ }^{9}$ Kronman (1995, p. 196) rastrea esta línea de pensamiento iniciada por Hobbes, a través de Chirstopher Langdell, el realismo jurídico, el análisis económico del derecho y los estudios críticos. En todos los casos el estudio del derecho es visto como una ciencia que debe encontrar alguna especie de principio oculto que subyace a la actividad práctica y particularista llevada adelante por los jueces.
} 
Pettit y los neo-republicanos piensan que es posible dirigir en contra de la libertad como no-interferencia un argumento análogo al que Berlín esgrime en contra de la libertad como no-frustración ${ }^{10}$. Si ser libre equivale a no sufrir interferencias, existe un modo contraintuitivo de alcanzar la libertad: congraciarse con quien posee el poder de interferencia, de manera que éste se comporte de modo amigable y se abstenga de ejercitar el poder que detenta. Si efectivamente el sujeto logra ganarse la buena voluntad del poderoso la probabilidad de interferencia será mínima y, de acuerdo con la concepción defendida por Berlín, el sujeto debería contar como libre. No obstante, pocos de nosotros sostendríamos que un sujeto en esta situación cuenta con libertad. Ser libre es estar protegido de la interferencia de terceros, aun en aquellos escenarios donde estos no son amigables.

De acuerdo con los neo-republicanos la protección frente a la interferencia de terceros debe extenderse no sólo a los escenarios donde éstos no poseen ningún deseo de interferir en las elecciones ajenas, sino también a aquellos donde tal deseo existe. La protección frente a la interferencia debe ser robusta tanto con relación a las preferencias y deseos del sujeto protegido - debe extenderse a escenarios donde éste posee preferencias diferentes a las actuales - como de los potenciales interferidores - debe extenderse a escenarios donde estos tienen el deseo o la preferencia por interferir - Mientras la libertad como no-interferencia es robusta sólo en el primer sentido, la libertad como no-dominación también lo es en el segundo.

Adicionalmente, la probabilidad de interferencia debe ser baja debido a la presencia de mecanismos institucionales. Según Pettit, «I cannot escape domination without the presence of protective institutions that testify to my non-domination. But I can escape interference, I can escape even the likelihood of interference, without the presence of such eloquent devices» (1997, p. 72). No sólo es importante que la interferencia sea improbable sino que es relevante también que los ciudadanos sean conscientes y estén seguros de que es así. Por esta razón los reaseguros institucionales establecidos a través del derecho son indispensables. La libertad es un tipo de status social del que gozamos en presencia de otros individuos gracias a la existencia del sistema jurídico y sus salvaguardas. A diferencia de la libertad como no-interferencia, la libertad como no-dominación no puede alcanzarse por el mero hecho de que no existan otros individuos, sino que requiere la existencia de otros y la presencia de mecanismos institucionales que limiten su poder de interferencia.

\footnotetext{
${ }^{10}$ La presentación que hago de las críticas republicanas a Berlin siguen lo que he señalado en otro lugar. Véase Seleme, 2015.
} 
Por último, los mecanismos institucionales que brindan protección contra la interferencia de terceros no deben encontrarse bajo el control de algún agente diferente al sujeto potencialmente interferido. Los ciudadanos son plenamente libres cuando las instituciones establecen barreras sobre el poder de interferencia incontrolado de terceros, y esto sólo puede darse si dichas instituciones se encuentran bajo el control del propio agente protegido (Pettit, 2012, pp. 50, 58, 170). Esto es así debido a la caracterización que Pettit ofrece de la no-dominación en términos de control. De acuerdo con esta definición «someone, A, will be dominated in a certain choice by another agent or agency, B, to the extent that B has a power of interfering in the choice that is not itself controlled by A» (Pettit, 2012, p. 50). De esto se sigue que si la capacidad de un agente A de interferir a otro $\mathrm{B}$ es controlada por un tercer agente $\mathrm{C}$-que impide el ejercicio de dicha capacidad - A no es libre sino que está dominado por C.

Para Pettit (2012, pp. 166-179) un agente posee control sobre otro cuando tiene la capacidad de influir sobre su curso de acción para dotarlo de una determinada dirección. El control es un tipo de influencia dirigida a un objetivo. A partir de esta noción, Pettit señala qué características debe tener el control que los ciudadanos ejercen sobre el aparato estatal para que sea cierto que éste se encuentra bajo el control de todos. El control debe ser individualizado, incondicionado y eficaz. El control es individualizado cuando cada ciudadano posee una capacidad de control que es igual a la que poseen los demás; es incondicionado cuando la influencia que cada ciudadano puede ejercitar no depende de la buena voluntad de otro; es eficaz cuando es tan efectivo para imprimir una dirección a las decisiones colectivas que cuando una decisión no es acorde con la dirección que un ciudadano deseaba imprimirle éste ve esta circunstancia meramente como una cuestión de mala suerte y no como la imposición de una voluntad ajena.

De esta manera, las instituciones de auto-gobierno pasan a ser instrumentos para alcanzar la libertad como no-dominación. Para que la influencia tenga una dirección definida, el sistema de toma de decisiones debe ser una democracia deliberativa representativa. Debe tratarse de un gobierno mixto, donde las agencias de gobierno se controlan recíprocamente evitando que alguna tenga un poder dominador. Finalmente, debe existir una ciudadanía contestataria cuya principal tarea es evaluar las decisiones colectivas.

De modo que Pettit (1997, pp. 17-18) presenta su concepción de libertad como no-dominación mostrando sus semejanzas y diferencias con la libertad negativa de Berlin. Estos acuerdos y desacuerdos se dan tanto a nivel normativo como institucional. A nivel normativo Pettit presenta un nuevo ideal de libertad con rasgos que la distinguen de la libertad como no- 
interferencia. A semejanza de ésta considera que para que los ciudadanos sean libres no es necesario que éstos lleven adelante ciertas acciones o desarrollen ciertos rasgos de carácter. Específicamente no equipara a la libertad con el auto-control personal (según el cual las partes inferiores del individuo son controladas por las superiores) o el auto-control colectivo (según el cual los individuos son libres cuando participan de la vida política de una comunidad política que a su vez se auto-gobierna). Los ciudadanos son libres meramente por estar protegidos frente al poder de interferencia incontrolado tanto privado como público. En esto la libertad como nodominación es tan negativa como la libertad como no interferencia. Se diferencia de ella en la extensión de la protección — que debe abarcar los escenarios donde los demás tienen el deseo de interferir-y los mecanismos de protección — que deben ser institucionales y encontrarse bajo el control de quienes son protegidos.

A nivel institucional Pettit (2012, p. 5) propone un sistema mixto de gobierno y la existencia de una ciudadanía contestataria que es diferente del diseño institucional propuesto por los defensores de la libertad como nointerferencia. La constitución mixta requiere la existencia de un estado de derecho y un orden constitucional en donde los ciudadanos gocen de iguales libertades y poderes, y donde ningún individuo o cuerpo tenga el control exclusivo sobre el derecho. La ciudadanía contestataria exige que los ciudadanos tengan la disposición de vigilar el funcionamiento de las instituciones públicas.

La primera consecuencia de adoptar a la libertad como no-dominación es que deja de ser cierta la idea de que toda norma jurídica interfiere con la libertad individual sin importar cuál haya sido su proceso de creación, la forma de gobierno en la que se enmarca, o su carácter legítimo o ilegítimo. Por el contrario, sin barreras institucionales que protejan a los ciudadanos del poder de interferencia de otros, que hayan sido establecidas por mecanismos democráticos que garanticen que no se encuentren bajo el control de terceros, la libertad como no-dominación es imposible. Aunque el derecho posee carácter coercitivo e interfiere con los deseos de los ciudadanos, si ha sido creado por mecanismos legítimos que otorgan el control del proceso a la ciudadanía y establece protecciones frente al poder de interferencia de otros, no representa ningún menoscabo a la libertad, sino que es su garante.

La tensión que existía entre las dos exigencias definitorias del rol de abogado si se interpretaba a la libertad domo no-interferencia (la de ser protectores de la libertad del cliente, defendiendo celosamente sus intereses, y la de actuar con apego a la ley promoviendo la realización de la justicia) desaparece. Si la libertad es un status social que se goza en el seno de un 
sistema jurídico que instala salvaguardas frente al poder de interferencia, cuyo control se encuentra en poder de la ciudadanía a través de mecanismos políticamente legítimos, proteger la integridad del sistema jurídico es equivalente a proteger la libertad del propio cliente.

Una vez que se concibe a la libertad como ausencia de dominación comienza a ser perceptible un nuevo rol de abogado. Las nuevas características que adquiere el rol profesional son las siguientes. Primero, el abogado deja de cumplir una tarea meramente técnica. Segundo, desaparece la confianza de que la mera defensa de los intereses del cliente contribuya a la realización del interés público. Tercero, la ética profesional pasa a estar centrada en las virtudes y la auto-regulación a través de los colegios profesionales adquiere una nueva justificación y sentido.

En primer lugar, dado que ya no es cierto que toda norma jurídica sea cual sea su procedimiento de creación- es igualmente costosa en términos de libertad, el rol del abogado como defensor de la libertad del cliente y promotor del interés público no puede ser meramente técnico. Puesto que el auto-gobierno colectivo ahora no representa una amenaza a la libertad corporizada en el intento de imponer medidas perfeccionistas, sino la única garantía de que los mecanismos institucionales no representen ellos mismos un poder dominador, es indispensable que el abogado se pregunte por los fines que persigue la norma jurídica y por la legitimidad del proceso que condujo a su creación.

El abogado no debe mostrar un respeto irrestricto a la ley, sea cual sea su origen, sino sólo a aquella que es fruto de un proceso democrático y deliberativo de toma de decisiones colectivas, en el seno de una constitución mixta donde existe un estado de derecho en el que los ciudadanos gozan de iguales libertades y poderes, y donde ningún individuo o cuerpo tiene el control exclusivo sobre el derecho. Dado que el cliente es libre sólo en tanto es ciudadano de un sistema jurídico con estas características, el abogado, en tanto defensor de su libertad, sólo debe respetar las leyes que forman parte de un sistema semejante.

Cuando las leyes carecen de legitimidad, la función del abogado pasa a ser de resistencia. Al oponerse a las leyes ilegítimas no sólo protege la libertad del cliente, sino que adicionalmente promueve el interés público. Ser "auxiliar de la justicia" deja de ser equivalente al mero "respeto por la legalidad". Si la ley es ilegítima — porque es un instrumento de dominación por parte de una facción que detenta el control sobre el aparato estatalpromover la justicia y defender la libertad del cliente no consiste en respetar la ley sino en enfrentarla.

Los sistemas jurídicos ilegítimos donde las barreras institucionales se encuentran bajo el control de unos pocos adoptan diferentes formas. En 
Latinoamérica el caso paradigmático han sido los entramados institucionales gestados por los golpes de Estado militares que azolaron la región en la segunda parte del siglo pasado. En Argentina, por ejemplo, el Estatuto para la Reorganización Nacional dictado en Marzo de 1976 por la junta militar que derrocó al gobierno democrático, fue la norma suprema del sistema jurídico — por encima de la Constitución que formalmente permaneció vigente - hasta el regreso de la democracia en $1983^{11}$. Los abogados que desconocieron la validez de este Estatuto e insistieron en la presentación de habeas corpus en beneficio del creciente número de personas desaparecidas por el terrorismo de Estado, son un ejemplo del tipo de resistencia que debe darse según el modelo republicano frente al derecho ilegítimo.

Cuando el sistema en general no es ilegítimo, tal como sucedía en el caso de las dictaduras militares referenciado en el párrafo previo, pero existen normas que persiguen fines que sí lo son, es necesario encontrar un modo de enfrentarlas que, a la vez, muestre respeto al orden legal legítimo. Una de las herramientas mejor calificadas para esta tarea es el litigio estratégico. Los abogados que aceptan litigar sobre ciertos casos como un modo de alcanzar reformas estructurales generales en relación con normas ilegítimas, son un ejemplo del modo en que la tarea de defender la libertad del cliente y la de ser auxiliar de la justicia son convergentes. El movimiento de litigio en interés público, originado en Estados Unidos durante la lucha por el reconocimiento de los derechos civiles en los años 60s, es un ejemplo de utilización de este tipo de herramienta en defensa de la libertad y enfrentando la ley ${ }^{12}$. Brown vs. Board of Education of Topeka es uno de sus casos paradigmáticos.

El abogado se comporta como defensor de la libertad del cliente y auxiliar de la justicia a través del mismo tipo de conducta: por promover los intereses del cliente que no son contrarios a las normas jurídicas legítimas, esto es a las creadas a través de un proceso democrático y deliberativo en el

\footnotetext{
${ }^{11}$ El art. 5 del Estatuto dejaba claro el carácter ilegítimo del nuevo sistema jurídico, al establecer que «las facultades legislativas que la Constitución Nacional otorga al Congreso, incluidas las que son privativas de cada una de las Cámaras, serán ejercidas por el Presidente de la Nación» que se encontraba en manos de las Fuerzas Armadas. El art. 9, por su parte, le otorgaba al presidente de facto el poder de designar a los miembros del poder judicial.

${ }^{12}$ El litigio estratégico es una herramienta neutra, en el sentido que puede ser utilizada para promover el acceso a ciertos derechos individuales y a la participación política, o puede ser utilizada en el sentido contrario. De allí que hablar de litigio estratégico y de litigio en interés público no es equivalente. En Argentina, por ejemplo, quienes más exitosamente han utilizado el litigio estratégico como herramienta han sido las ONG opuestas al aborto y a ciertas prácticas anticonceptivas (Peñas Defago \& Morán Faúndes, 2014). Por otro lado, un análisis de cómo el litigio estratégico puede utilizarse para promover los derechos humanos en el campo de la salud, puede encontrarse en Talbot (2013).
} 
marco de una constitución que garantiza la igualdad de derechos y poderes por parte de la ciudadanía. Para hacerlo, indefectiblemente debe preguntarse por los fines que persigue la norma jurídica y el proceso que le dio origen, al igual que debe evaluar los intereses que su propio cliente quiere satisfacer. Su rol no es el de un mero técnico que pone sus habilidades jurídicas al servicio de quien lo contrata despreocupándose por cuáles son los fines que éste persigue.

En segundo lugar, desaparece la confianza de que la defensa de cualquier interés del cliente que encaje con la letra de la ley contribuirá a la promoción de la justicia. La "celosa defensa dentro de los límites de la ley" deja de ser la idea rectora de la profesión de la abogacía. El abogado sólo debe respetar y salvaguardar las leyes que son legítimas. Adicionalmente, no debe defender la libertad de su cliente intentando disminuir el impacto que las normas jurídicas legítimas puedan tener sobre la satisfacción de sus intereses. Si el abogado brinda una interpretación de la norma jurídica que es contraria a los fines legítimos que ésta persigue, de modo que no interfiera con los intereses de su cliente, no ha satisfecho ni la exigencia de promover su libertad ni la exigencia de ser "auxiliar de la justicia" y promover el interés público. Esto porque la libertad individual como no-dominación y el auto-gobierno colectivo no son independientes.

Cuando el auto-gobierno pasa a ser valorado como un instrumento que busca evitar que los mecanismos de protección frente al poder de interferencia de terceros no se encuentren bajo el control exclusivo de un agente, dispersando su control en toda la ciudadanía, el potencial tiránico que Berlin veía en el mismo desaparece. El auto-gobierno deja de aparecer como una prolongación a nivel colectivo de la libertad como auto-control individual, que busca someter las partes inferiores de uno mismo a las superiores. El auto-control colectivo deja de ser visto como un intento de que las partes más valiosas de la sociedad se impongan sobre las menos valiosas, a través de políticas perfeccionistas, y pasa a ser considerado como un instrumento para garantizar exactamente lo contrario, esto es la dispersión del control.

La misma idea de evitar la dominación a través de la dispersión del control brinda una nueva justificación al sistema adversarial. Que las partes a quienes se va a aplicar la norma jurídica individual contenida en la sentencia, puedan tener una función activa en su proceso de creación (v.gr. ofreciendo prueba, diligenciándola, presentando hipótesis explicativas de los hechos, alegando a favor de su pretensión, etc.) garantiza que ningún tercero ejerza sobre ellas el control. La tarea del abogado es ayudar a que el cliente pueda ejercitar de manera eficiente el control sobre el proceso judicial. La división del trabajo, haciendo que cada abogado se focalice en 
su propio cliente, es una herramienta para lograr que el control se encuentre disperso. Cada abogado actúa como un "igualador retórico" que equilibra la diferente capacidad argumentativa que poseen los individuos que quieren hacer valer sus pretensiones en el proceso ${ }^{13}$.

Ver al sistema adversarial como una herramienta para evitar la dominación hace que las reglas procesales, y la exigencia de "celosa defensa" que lo caracteriza, deban ser vistas a una nueva luz. Lo que justifica que el abogado se concentre en lograr que su cliente tenga el control sobre el proceso es la certeza de que otro abogado está intentando lograr lo mismo en relación con la contraparte. No puede ser de otro modo si la justificación última del sistema es lograr la dispersión del control. Si este es el caso, las estrategias utilizadas por los abogados para entorpecer el control que la contraparte ejercita sobre el proceso, y la despreocupación por el hecho de que el abogado que la asiste está cumpliendo eficazmente su rol, se encuentran injustificadas.

Las estrategias de la "lista de preguntas sucias"14, greymailing ${ }^{15}$, y las demandas estratégicas en contra de la participación pública ${ }^{16}$ son ejemplos del primer tipo de conducta indebida. Lo que intenta el abogado que las utiliza es impedir que la contraparte pueda ejercitar sus herramientas de control sobre el proceso, en el caso extremo intimidándola para retirar o no presentar la demanda. El abogado actúa como un instrumento de dominación que no sólo socava la libertad de la contraria, sino que también ayuda a

${ }^{13}$ La expresión “igualadores retóricos” también pertenece a Böhmer (2008).

${ }^{14} \mathrm{El}$ abogado pregunta a la contraparte una lista de preguntas que la obligan a revelar algunos hechos íntimos o vergonzosos con la excusa de que pueden tener relevancia para la causa. Cuando se está enfrentando un juicio al que le pueden seguir otros con el mismo objeto por diferentes litigantes, el mensaje que se les envía a estos es que deberán enfrentarse al mismo proceso vergonzante si deciden accionar.

${ }^{15}$ El nombre deriva de lo semejante que es a extorsionar (en inglés blackmailing). La estrategia fue utilizada por los abogados defensores del ex-director de la CIA, Richard Helms. El funcionario había mentido acerca del involucramiento de la agencia de inteligencia en el derrocamiento del presidente chileno Salvador Allende. Para evitar su enjuiciamiento sus abogados defensores exigieron la desclasificación de una serie de expedientes en los que se encontraba contenida información que podía dañar, o al menos comprometer, al gobierno estadounidense. El objetivo de la táctica era presionar al gobierno para que desistiese del intento de enjuiciamiento

16 Se trata de una estrategia utilizada usualmente por las grandes corporaciones o por clientes con cuantiosos recursos económicos. Aquí los ciudadanos que están protestando en contra de alguna medida llevada adelante por la empresa o corporación son demandados, generalmente por difamación. Aunque la demanda por difamación no tenga ningún fundamento, tiene un efecto disuasorio sobre cualquier que en el futuro intente protestar. El mensaje es que tendrá que enfrentar los costos personales y económicos que implica un proceso judicial. 
erosionar las reglas procesales que confieren a su propio cliente el estatus de ciudadano libre del poder de interferencia arbitrario.

$\mathrm{El}$ aprovechamiento de la negligencia en la defensa en la que incurre el abogado de la contraria es un ejemplo del segundo defecto. El abogado que espera sorprender a la contraria, por ejemplo, pidiendo la perención de instancia sin asegurarse de que el plazo no se ha vencido por mera negligencia del letrado de la contraparte, no puede justificar su "celosa defensa" en la certeza de que otro está cumpliendo su mismo rol en relación con la contraria. La práctica de avisar a la contraria antes de pedir la perención no es un rasgo de cortesía entre colegas, sino que hunde sus raíces en la justificación republicana del sistema adversarial.

Adicionalmente, la idea de libertad como no-dominación determina que el abogado deba evaluar los fines y deseos de su cliente y no sólo oficiar como un instrumento para su satisfacción. La tarea del abogado cuando el cliente busca frustrar los fines que persiguen las normas jurídicas legítimas -que establecen la infraestructura que protege a los ciudadanos en contra del poder de interferencia arbitrario- consiste en mantener su independencia de criterio y actuar como elemento de contención en lugar de facilitador ${ }^{17}$. El abogado debe involucrarse activamente en evaluar y modelar los intereses del cliente haciendo que estos no entren en conflicto con los fines que persiguen las normas jurídicas legítimas. Al hacerlo defiende la libertad del cliente que sólo se encuentra asegurada por las normas jurídicas legítimas que le confieren su estatus de seguridad frente al poder de interferencia incontrolado.

A semejanza de lo que sucedía en la concepción hobbesiana como nofrustración el abogado puede promover la libertad de su cliente alterando sus deseos. A diferencia de lo que sucedía allí, no se trata meramente de hacer que modifique aquellos deseos que se encuentran bloqueados por la existencia de normas jurídicas coercitivas. Para la libertad como nofrustración sólo importaba tener deseos cuya satisfacción se encuentre libre de interferencia. Por el contrario, para la libertad como no-dominación los deseos que deben ser extinguidos para proteger la libertad son aquellos contrarios a las normas jurídicas legítimas que establecen las salvaguardas frente al poder de interferencia incontrolado de otros.

\footnotetext{
${ }^{17}$ Robert W. Gordon (1988) ha explorado el carácter independiente que deben tener los abogados si se adopta el paradigma republicano de libertad. No sólo ha llamado la atención sobre la necesidad de que los abogados sean parcialmente independientes en relación con los intereses del cliente, sino también las condiciones institucionales y sociales que deben darse para que la independencia pueda tener cabida.
} 
En este respecto la diferencia con el rol de abogado que surge de la libertad como no-interferencia no podría ser mayor. Si bien de acuerdo con ambos modos de concebir la profesión de abogado éste no debe promover los deseos ilegales del cliente, si se adopta la libertad como no-dominación, no promover algunos deseos del cliente puede ser una manera de proteger su libertad. Para la libertad como no-interferencia que el abogado intente alterar los deseos del cliente no es un requerimiento que se siga de la exigencia de proteger su libertad. Para la libertad como no-dominación si las normas jurídicas son legítimas, oponerse a la satisfacción de los deseos ilegales del cliente es un modo de promover su libertad.

En tercer lugar, concebir a la libertad como no-dominación conduce a una manera de entender las regulaciones profesionales existentes. Dado que las normas jurídicas deben ser interpretadas de acuerdo con los fines legítimos que persiguen, y no se debe intentar minimizar a toda costa su poder de interferencia sobre los deseos que posee el cliente, una estrategia interpretativa semejante se aplica sobre los estándares normativos que regulan la conducta profesional. Así como el buen abogado no es aquel que intenta minimizar el poder de interferencia de las normas jurídicas legítimas sobre las conductas del cliente, los buenos departamentos de ética profesional con los que cuentan los estudios jurídicos no son aquellos que intentan minimizar la interferencia de los estándares éticos sobre la conducta de los abogados. El buen abogado es aquel que interpreta los estándares éticos de acuerdo con sus fines y no busca frustrarlos en el ejercicio de la profesión.

Adicionalmente, la resistencia de los abogados frente a los intentos estatales de regular la profesión, encuentra sustento también en la libertad como no-dominación. De acuerdo con esta concepción existen dos tipos de dominación que deben ser evitadas: la dominación horizontal entre ciudadanos y la dominación vertical ejercitada por el Estado ${ }^{18}$. Parte de la tarea del abogado en tanto defensor de la libertad de su cliente consiste en impedir que el Estado ejerza este poder dominador. Adicionalmente, el abogado debe realizar esta tarea de salvaguarda sin volverse él mismo un instrumento de dominación. Si el abogado impide que otros ciudadanos o el propio Estado ejerza sobre su cliente un poder incontrolado, pero lo hace a través de estrategias sobre las que el cliente no ejerce ningún control — por

\footnotetext{
${ }^{18}$ De acuerdo con Pettit (2012, p. 3), la legitimidad se refiere a los estándares de corrección que se aplican a las relaciones que existen entre los ciudadanos y su Estado. Los criterios de justicia, por el contrario, se aplican a las relaciones que los ciudadanos tienen entre sí. Un Estado es legítimo cuando el poder que ejercita se encuentra bajo el control de la ciudadanía. Es justo, cuando impide que los ciudadanos ejerciten unos sobre otros un poder incontrolado.
} 
ejemplo, porque no las ha consentido o ni siquiera las conoce - entonces la libertad republicana del cliente se encuentra amenazada.

La auto-regulación ejercitada a través de los colegios profesionales se presenta como una herramienta apta para alcanzar estos fines. En primer lugar, las asociaciones profesionales articulan el control de pares sobre los abogados para evitar que estos ejerzan sobre sus clientes un poder incontrolado. Un mecanismo que se presenta como especialmente apto para alcanzar este fin — si se adopta la concepción de libertad como nodominación - es el de los Códigos de Honor asentados en la tradición y la costumbre, fundados en la promoción de la virtud y el castigo a través de la deshonra. Este tipo de control es diferente al que se ejercita a través del derecho penal disciplinario. Mientras el poder disciplinario es uno de carácter estatal que ejercita el control de la profesión desde fuera, para utilizar una metáfora, el control a través del honor es desde dentro y es ejercido por los pares.

Dos razones confluyen para que el control a través de la virtud y el honor sea atractivo si se concibe a la libertad como no-dominación. La primera, se refiere a la idea neo-republicana según la cual para que no exista dominación deben existir salvaguardas institucionales contra el poder de dominación sobre las que nadie ejercite el control. La dispersión del control sobre los mecanismos institucionales es sólo un medio para que nadie lo ejercite de manera exclusiva. Es un medio que garantiza no estar sometido a la voluntad de otro. Esto dota de un atractivo especial a las normas de origen consuetudinario sobre las que nadie posee el control deliberado de creación o derogación. Mientras más inmune sea al cambio deliberado el sistema de normas consuetudinarias que evitan que los abogados ejerciten un poder de interferencia incontrolado sobre los clientes, más se acercan a lo que Pettit denomina «an impersonal, will-independent regime of protection» $(2012$, p. 134) frente a la dominación.

La segunda, tiene que ver con las peculiaridades de la relación abogado-cliente que hacen que sea difícil controlarla de manera externa. $\mathrm{La}$ exigencia de que la relación esté protegida por el deber de secreto o sigilo, por ejemplo, determina que las posibilidades de control por parte de los pares o por parte del propio abogado sean mucho mayores. Un cuerpo de abogados dotados de virtud cívica aparece como una herramienta inmejorable para alcanzar esta tarea. La virtud cívica posee tres elementos. En primer lugar, quienes la poseen respetan las normas por sí mismas y no meramente por temor al castigo y, en consecuencia, no intentan evadirlas a la primera oportunidad de hacerlo. Esto, adicionalmente, garantiza que las normas no sean utilizadas como meros instrumentos para promover fines particulares. Por último, quienes poseen este tipo de virtud ejercitan una constante 
vigilancia intentando evitar la aparición de poderes incontrolados (Pettit, 1997, pp. 246-251). Resaltando la importancia de este tipo de control ejercitado a través de normas consuetudinarias que no son impuestas por el aparato estatal, Pettit señala: «the state must be very careful not to introduce heavy-handed patterns of control that are likely to undermine the influence of this more or less autonomous mode of regulation» (p. 254).

Esta idea de abogado virtuoso - entendido como aquel que acepta los estándares normativos que regulan el ejercicio profesional, no los tergiversa utilizándolos como armas en beneficio propio, y tiene una actitud vigilante sobre los demás - se aparta de los ideales de virtud de tinte perfeccionista que preocupaban a los defensores de la libertad como no-interferencia. Aunque hace referencia a rasgos de carácter que deben ser cultivados y desarrollados, se trata de un ideal de virtud cívica vinculado sólo con la aceptación de estándares que se aplican a la conducta profesional.

El auto-control de la profesión ejercitado a través de las asociaciones profesionales también sirve para garantizar que los abogados actúen como freno al poder incontrolado del Estado. Si se permite que el Estado regule el ejercicio del profesional a través de su poder disciplinario, es posible que éste utilice dicho poder para hostigar a los abogados que están intentando proteger a los ciudadanos de los intentos estatales de ejercitar un poder dominador ilegítimo. Ante este riesgo, la idea de regular la profesión a través del honor y la virtud se presenta como atractiva ${ }^{19}$.

La colegiación de los abogados en asociaciones no solo permite sortear la regulación estatal de la profesión sino que, adicionalmente, ayuda a fortalecer la posición de los abogados como salvaguardas frente al poder arbitrario del estado. Si el abogado, en tanto defensor de la libertad de su cliente, tiene que actuar como una especie de freno o contrapeso al poder dominador del Estado, las perspectivas no parecen demasiado prometedoras. Frente al colosal poder de interferencia estatal se erige un único profesional como primera barrera. La colegiatura viene a compensar, aunque sea parcialmente, esta desventaja. El Colegio de Abogados funciona como un reaseguro de que el Estado no perseguirá u hostigará a los profesionales porque detrás de cada abogado, por así decirlo, ahora están todos.

\footnotetext{
${ }^{19}$ John M. Najemy (1979) ha llamado la atención sobre un tipo de Republicanismo surgido en Florencia en el Siglo XIV cuyo componente primigenio no eran los ciudadanos vistos de manera aislada sino los gremios profesionales. Aunque el gremio de los abogados no apareció durante toda la Edad Media — debido al hecho de que el abogado era equiparado a un oficial público - la existencia de este "guild Republicanism" ayuda a percibir los vínculos que pueden trazarse entre la colegiatura de la profesión de abogado y la justificación republicana de las instituciones públicas.
} 


\section{CONCLUSIÓN}

El carácter defectuoso que posee la concepción de la libertad como nointerferencia - puesto de manifiesto por las críticas neo-republicanashace que sea igualmente defectuoso el rol profesional que se funda sobre ella. Cuando esta noción defectuosa de libertad es reemplazada por la libertad como no-dominación republicana el modo de concebir la profesión de abogado adquiere nuevos contornos.

En primer lugar, es posible reconciliar la exigencia que pesa sobre el abogado de proteger la libertad del cliente con su deber de actuar como un auxiliar de la justicia. La preocupación por mantener incólume el aparato institucional estatal, que garantiza que el cliente esté libre del poder de interferencia arbitrario ejercitado por los particulares o por el propio Estado, es congruente con la preocupación por proteger su libertad individual. Para hacerlo el abogado debe preguntarse indefectiblemente por los fines que persiguen las normas jurídicas y por su modo legítimo de origen.

En segundo lugar, y vinculado con lo anterior, el abogado deja de ser un mero asistente técnico que busca encontrar cualquier resquicio en el entramado legal que permita que el cliente satisfaga sus intereses, sean estos cuales sean, y pasa a ser la primera barrera que se opone a la satisfacción de aquellos intereses que, aunque acordes con la letra de la ley, intentan frustrar sus fines.

En tercer lugar, el sistema adversarial pasa a ser un instrumento para dispersar el control sobre todas las partes involucradas en el proceso, garantizando que la norma particular contenida en la sentencia no se transforme en un instrumento de dominación. Cada abogado puede concentrarse en garantizar que su representado tenga el control sobre el proceso, bajo la condición de que existe otro abogado intentando hacer lo mismo con la contraparte.

En cuarto lugar, la autorregulación a través de los colegios profesionales pasa a ser un modo de prevenir tanto la dominación horizontal como la vertical sobre los abogados. Que este control se ejercite a través de normas consuetudinarias de difícil modificación, aceptadas e impuestas por los propios abogados, garantiza que éstas no se encuentren bajo el control particular de nadie, ni de un grupo de profesionales ni del propio Estado. 


\section{REFERENCIAS}

Annas, J. (2011). Intelligent Virtue. New York: Oxford University Press.

Berlin, I. (1969). Two Concepts of Liberty. In Four Essays on Liberty (pp. 118-172). Oxford: Oxford University Press.

Berlin, I. (2002). Two Concepts of Liberty. In Liberty (pp. 166-217). Oxford: Oxford University Press.

Böhmer, M. (2008). Igualadores y Traductores. La Ética del Abogado en una Democracia Constitucional. In M. Alegre, R. Gargarella, \& C. Rosenkrantz (eds.), Homenaje a Carlos Nino. Buenos Aires: La Ley.

Chambliss, E., \& Wilkinks, D. (2002). Promoting Ethical Infrastructure in Large Law Firms: A Call for Research and Reporting. Hofstra Law Review, 30(3), pp. 691716.

Chappell, T. (2014). Knowing What to Do. Oxford: Oxford University Press.

Finnis, J. (1996). The Truth in Legal Positivism. En R. P. George (ed.), The Autonomy of Law: Essays on Legal Positivism (pp. 195-214). Oxford: Oxford University Press.

Gordon, R. W. (1988). The Independence of Lawyers. Boston University Law Review, 68(1), pp. 1-83.

Hayek, F. (1960). The Constitution of Liberty. London: Routledge \& Keegan Paul.

Hayek, F. (1973). Law, Liberty and Legislation, vol. I. Chicago: University of Chicago Press.

Hobbes, T. (1997). Leviathan. New York: Touchstone.

Koniak, S. P., \& Cohen, G. M. (2000). In Hell There Will Be Lawyers Without Clients or Law. In D. L. Rhode (ed.), Ethics in Practice: Lawyer's Role, Responsibility and Regulation (pp. 177-204). Oxford: Oxford University Press.

Kronman, A. (1995). The Last Lawyer: Failing ideals of the Legal Profession. Cambridge, Massachusetts: Harvard University Press.

Laborde, C. (2008). Critical Republicanism: The Hijab Controversy and Political Philosophy. Oxford: Oxford University Press.

Lovett, F. (2001). Domination: A preliminary analysis. The Monist, 84, pp. 98-112.

Lovett, F. (2010). A General Theory of Domination and Justice. Oxford: Oxford University Press.

Lovett, F. (2012). What Count as Arbitrary Power? Journal of Political Power, 5(1), pp. $137-152$.

Martí, J. luis. (2006). La República Deliberativa. Madrid-Barcelona: Marcial Pons.

Maynor, J. (2003). Republicanism in the Modern World. Cambridge: Polity Press. 
Najemy, J. M. (1979). Guild Republicanism in Trecento Florence: The Successes and Ultimate Failure of CorporatePolitics. The American Historical Review, 84(1), pp. 53-71.

Peñas Defago, M. A., \& Morán Faúndes, J. M. (2014). Conservative litigation against sexual and reproductive health policies in Argentina. Reproductive Health Matters, 22(44), pp. 82-90.

Pettit, P. (1997). Republicanism: A Theory of Freedom and Government. Oxford: Clarendon Press.

Pettit, P. (2011). The Instability of Freedom as Noninterference: The Case of Isaiah Berlin. Ethics, 121 , pp. 693-716.

Pettit, P. (2012). On The People's Terms. Cambridge: Cambridge University Press.

Seleme, H. O. (2015). Un Nuevo Adversario de la Libertad como No-Dominación. Araucaria. Revista Iberoamericana de Filosofía Política y Humanidades, 33, pp. 59-82.

Skinner, Q. (1995). The Paradoxes of Liberty. In S. Darwall (Ed.), Equal Freedom (pp. 1538). Michigan: The University of Michigan Press.

Slote, M. (2001). Morals from Motives. Oxford: Oxford University Press.

Swanton, C. (2003). Virtue Ethics: A Pluralistic View. Oxford: Oxford University Press.

Talbot, S. (2013). Advancing human rights in patient care through strategic litigation: Challenging medical confidentiality issues in countries in transition. Health and Human Rights, 15(2), pp. 69-79.

Viroli, M. (2002). Republicanism. New York: Hill and Wang.

Zagzebski, L. (2010). Exemplarist Virtue Theory. Metaphilosophy, 41(1), pp. 41-57. 